\title{
Is metabolic syndrome associated with high tumor grade and stage of bladder cancer: a systematic review and meta-analysis
}

\author{
Dechao Feng ${ }^{1 \#}$, Pan Song ${ }^{1 \#}$, Yubo Yang ${ }^{1 \wedge}$, Wuran Wei ${ }^{1 \wedge}, \mathrm{Li} \mathrm{Li}^{2,3}$ \\ ${ }^{1}$ Department of Urology, Institute of Urology, West China Hospital, Sichuan University, Chengdu, China; ${ }^{2}$ Department of Pediatrics, West China \\ Second University Hospital, Sichuan University, Chengdu, China; ${ }^{3}$ Key Laboratory of Birth Defects and Related Diseases of Women and Children \\ (Sichuan University), Ministry of Education, Chengdu, China \\ Contributions: (I) Conception and design: D Feng; (II) Administrative support: W Wei, L Li; (III) Provision of study materials or patients: D Feng, P \\ Song, Y Yang; (IV) Collection and assembly of data: W Wei, Y Yang; (V) Data analysis and interpretation: D Feng, P Song; (VI) Manuscript writing: \\ All authors; (VII) Final approval of manuscript: All authors. \\ \#These authors contributed equally to this work. \\ Correspondence to: Li Li. Department of Pediatrics, West China Second University Hospital, Sichuan University, No. 20, Section 3, Renmin Nan Lu, \\ Chengdu 610041, China. Email: liliwch@126.com.
}

Background: The aim of our study was to elaborate the association between metabolic syndrome (MS) and the tumor stage and grade of bladder cancer (BC).

Methods: A systematic review and pooled analysis on PubMed, the Cochrane Library, Embase, Web of Science, CNKI, WANFANG and VIP from databases inception to July 24, 2020 was conducted by two independent authors. Relative risk (RR) was used as pooled effect estimates. The data analysis was accomplished by STATA 14.2.

Results: Three English and four Chinese articles were included in the final analysis. A pooled analysis of six studies showed that patients in MS group were at a 1.94-fold risk of high-stage BC when compared to their counterparts (RR: 1.94; 95\% CI: 1.59-2.37), and the difference was statistically significant. For the components of MS, except for hypertension, patients with obesity (RR: 1.61; 95\% CI: 1.33-1.95), hyperglycemia (RR: 2.20; 95\% CI: 1.49-3.26) and low high-density lipoprotein cholesterol (HDL) (RR: 1.98; 95\% CI: 1.51-2.58) had significantly higher risks of high-stage BC than the control groups. A pooled analysis of six studies indicated that MS can contribute substantially to the vulnerability of high-grade BC with significant difference (RR: 1.50; 95\% CI: 1.37-1.65). Furthermore, patients with obesity (RR: 1.41; 95\% CI: 1.18-1.69), hyperglycemia (RR: 1.42; 95\% CI: 1.30-1.56), hypertension (RR: 1.13 ; 95\% CI: 1.03-1.24), low HDL (RR: 1.29; 95\% CI: 1.14-1.46) and high triglyceride (TG) (RR: 1.28; 95\% CI: 1.11-1.46) were at a higher risk of high-grade $\mathrm{BC}$ than their counterparts.

Conclusions: This meta-analysis revealed that MS and its components might be associated with high BC stage and grade.

Keywords: Metabolic syndrome (MS); bladder cancer (BC); stage; grade; meta-analysis

Submitted Nov 30, 2020. Accepted for publication Mar 26, 2021.

doi: $10.21037 /$ tcr-20-3350

View this article at: http://dx.doi.org/10.21037/tcr-20-3350

\footnotetext{
^ ORCID: Dechao Feng, 0000-0002-8267-9920; Yubo Yang, 0000-0002-0189-3256; Wuran Wei, 0000-0002-2133-6043.
} 


\section{Introduction}

Bladder cancer (BC) is one of the most common fatal urological tumors in the world, with an estimated 573,000 new cases and 213,000 death yearly (1). Regardless of gender, the incidence of BC in China has increased year by year in both urban and rural areas (2), which not only negatively affected the patients' quality of life but also increased the economic burden on families and the national health care system (3). Thus, enough attention should be paid to the management of $\mathrm{BC}$.

$\mathrm{BC}$ is multifactorial in origin, including internal genetic factors and external environmental factors, and the mechanism responsible for this condition is unclear. Cigarette smoking and occupational exposure are two relatively clear risk factors of $\mathrm{BC}$, and the carcinogenesis of these pathogenic factors is likely to be associated with the presence of aromatic amines, such as 2-naphthylamine, 4-aminobiphenyl and 4-chloro-ortho-toluidine (4). The other controversial factors include urinary tract infection $(5,6)$ and metabolic syndrome (MS) (7). MS is multifactorial condition, characterized by overweight or obesity, hypertension, hyperglycemia, and dyslipidemia. In recent years, MS has become an emphatic public health problem due to its close association with a further increase of risk of cardiovascular diseases and type 2 diabetes, and its prevalence is increasing (8-11). It is notable that MS has been demonstrated as a possible predisposing factor for the development and progression of various malignant tumors, including renal cell cancer, live cancer, oesophageal cancer, pancreatic cancer, prostate cancer and bladder cancer $(7,12,13)$. However, there are limited data so far reporting the correlations either between MS and the tumor stage and grade of BC. In such situations, we performed a systematic review and meta-analysis to explore these potential relationships. We present the following article in accordance with the PRISMA reporting checklist (available at http://dx.doi.org/10.21037/tcr-20-3350).

\section{Methods}

\section{Study selection}

Electronic literature search of seven domestic and foreign databases and manual search of bibliographies of key retrieved articles and relevant reviews were conducted systematically without language restrictions in accordance with the Preferred Reporting Items for Systematic Review and Meta-analyses guidelines (14) from inception to July
24, 2020. The English databases included PubMed, the Cochrane Library, Embase and Web of Science, and the Chinese databases involved CNKI, WANFANG and VIP. The searched terms or keywords related to this article included "Metabolic Syndrome" and "Urinary Bladder Neoplasms", and the detailed strategy used in PubMed was as follows: $(()(()((()(()(()(()($ Urinary Bladder Neoplasms[Title/Abstract]) OR (Neoplasm, Urinary Bladder[Title/Abstract])) OR (Urinary Bladder Neoplasm[Title/Abstract])) OR (Neoplasms, Bladder[Title/Abstract])) OR (Bladder Neoplasms[Title/ Abstract])) OR (Bladder Neoplasm[Title/Abstract])) OR (Neoplasm, Bladder[Title/Abstract])) OR (Bladder Tumors[Title/Abstract])) OR (Bladder Tumor[Title/ Abstract])) OR (Tumor, Bladder[Title/Abstract])) OR (Tumors, Bladder[Title/Abstract])) OR (Urinary Bladder Cancer[Title/Abstract]))) OR (Cancer, Urinary Bladder[Title/Abstract])) OR (Malignant Tumor of Urinary Bladder[Title/Abstract])) OR (Cancer of the Bladder[Title/ Abstract])) OR (Bladder Cancer[Title/Abstract])) OR (Bladder Cancers[Title/Abstract])) OR (Cancer, Bladder[Title/Abstract])) OR (Cancer of Bladder[Title/

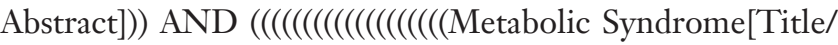
Abstract]) OR (Metabolic Syndromes[Title/Abstract])) OR (Syndrome, Metabolic[Title/Abstract])) OR (Syndromes, Metabolic[Title/Abstract])) OR (Metabolic Syndrome $\mathrm{X}$ [Title/Abstract])) OR (Insulin Resistance Syndrome X[Title/Abstract])) OR (Syndrome X, Metabolic[Title/ Abstract])) OR (Syndrome X, Insulin Resistance[Title/ Abstract])) OR (Metabolic X Syndrome[Title/Abstract])) OR (Syndrome, Metabolic X[Title/Abstract])) OR (X Syndrome, Metabolic[Title/Abstract])) OR (Dysmetabolic Syndrome X[Title/Abstract])) OR (Syndrome X, Dysmetabolic[Title/Abstract])) OR (Reaven Syndrome X[Title/Abstract])) OR (Syndrome X, Reaven[Title/ Abstract])) OR (Metabolic Cardiovascular Syndrome[Title/ Abstract])) OR (Cardiovascular Syndrome, Metabolic[Title/ Abstract])) OR (Cardiovascular Syndromes, Metabolic[Title/Abstract])) OR (Syndrome, Metabolic Cardiovascular[Title/Abstract])). The specific strategy is showed in Supplementary material. All observational studies reporting the following outcomes of interest were pooled and analyzed. Two authors independently checked and reviewed the titles and abstracts of identified studies. Then, the articles meeting the inclusion criteria were searched for further evaluation, and data were extracted independently by two reviewers. Disagreements were settled by discussion or a third party. We extracted the following data in accordance 
with a predetermined table: (I) the first author and year of publication; (II) study design; (III) the characteristics of the recruited patients, and (IV) percentages of upstage and upgrade.

\section{Selection criteria}

We used the following PICOS method to determine the eligibility of included studies: Patients $(\mathrm{P})$ : patients diagnosed with primary BC; Intervention (I): MS patients who satisfied at least three of the following four criteria: (I) overweight or obesity (BMI $\geq 25 \mathrm{~kg} / \mathrm{m}^{2}$ ); (II) blood pressure $(\mathrm{BP})>135 / 85 \mathrm{mmHg}$ or undergoing treatment for hypertension; (III) fasting plasma glucose $\geq 6.1 \mathrm{mmol} / \mathrm{L}$ or 2-hour plasma glucose $\geq 7.8 \mathrm{mmol} / \mathrm{L}$ or undergoing treatment for hyperglycemia; (IV) dyslipidemia: triglyceride (TG) $\geq 1.70 \mathrm{mmol} / \mathrm{L}$ or high-density lipoprotein cholesterol (HDL) $<0.9 \mathrm{mmol} / \mathrm{L}$ in males and $>1.0 \mathrm{mmol} / \mathrm{L}$ in females, or undergoing treatment for high TG or low HDL; Comparison (C): studies comparing MS group to nonMS group; Outcomes $(\mathrm{O})$ : $\mathrm{BC}$ stage and grade; Ta and $\mathrm{T} 1$ tumors were defined as lower stage BC. T2, T3 and T4 tumors were defined as higher stage $\mathrm{BC}$; The pathological grade was determined based on the 2004 World Health Organization grading system. Study design (S): observation studies published in full text. For articles which might include the same population, we only enrolled the largest one unless different outcomes of interest were reported.

\section{Quality assessment}

We used the Newcastle-Ottawa Quality Assessment Scale to assess the quality of the observational studies (15). This tool evaluates the case-control studies from three domains: selection, comparability, and exposure. Each study can be given a maximum of one star for each numbered item within the selection and exposure categories, and a maximum of two stars can be given for comparability. The rated studies with seven or more stars were considered as high quality. Additionally, two authors independently evaluated the level of evidence of included articles according to the Oxford Centre for Evidence-Based Medicine criteria (16). This scale rated studies from strongest (level 1) to weakest (level 5) strength of evidence on the basis of study design and data quality.

\section{Statistical analysis}

The dichotomous variables were presented as proportions and corresponding $95 \%$ confidence intervals (CIs). Relative risk (RR) was estimated of for dichotomous variables. The Cochran Q test (17) and $\mathrm{I}^{2}$ test (18) were used to assess heterogeneity among studies, and $\mathrm{I}^{2}>50 \%$ was seemed to be significant. The random effects model was used when heterogeneity was yielded $(\mathrm{P}<0.1)$, otherwise the fixed effects model was used. Sensitivity analysis was conducted to determine the source of heterogeneity. A subgroup analysis of Chinese studies was conducted due to ethnicity of included studies. Statistical significance was regarded as $\mathrm{P}<0.05$. The data analysis was accomplished by STATA 14.2.

\section{Results}

\section{Literature search results}

We searched PubMed, Embase, the Cochrane Library, Web of Science and three other Chinese databases for subject headings and text words related to "Metabolic Syndrome" and "Urinary Bladder Neoplasms" in articles published from inception to July 24, 2020 without language restrictions. Besides, we also conducted a manual search of references of retained articles and previous reviews to pledge a comprehensive retrieve. After excluding duplicates and screening titles and abstracts, seven studies were potentially eligible. Eventually, three English (19-21) and four Chinese (22-25) articles were enrolled in the analysis. The flow chart of study selection was showed in Figure 1. A total of seven articles (19-25) evaluated the association of MS and its components with tumor stage and grade of bladder cancer. Of the seven included studies, five are from China (19,22-25), one from Japan (21), and one from Turkey (20). Table 1 presents the basic characteristic of the included studies.

\section{MS and BC stage}

A pooled analysis of six studies (19,21-25) with a total of 1,311 participants showed that patients in MS group were at a 1.94-fold risk of high-stage $\mathrm{BC}$ when compared to their counterparts (RR: 1.94; 95\% CI: 1.59-2.37) (Figure 2), and the difference was statistically significant. For the components of MS, except for hypertension, patients with obesity, hyperglycemia and Low HDL had significantly higher risks of high-stage $\mathrm{BC}$ than the control groups, and the corresponding RRs and 95\% CIs were $1.61(1.33,1.95)$, $2.20(1.49,3.26)$ and $1.98(1.51,2.58)$, respectively (Figure 2). 

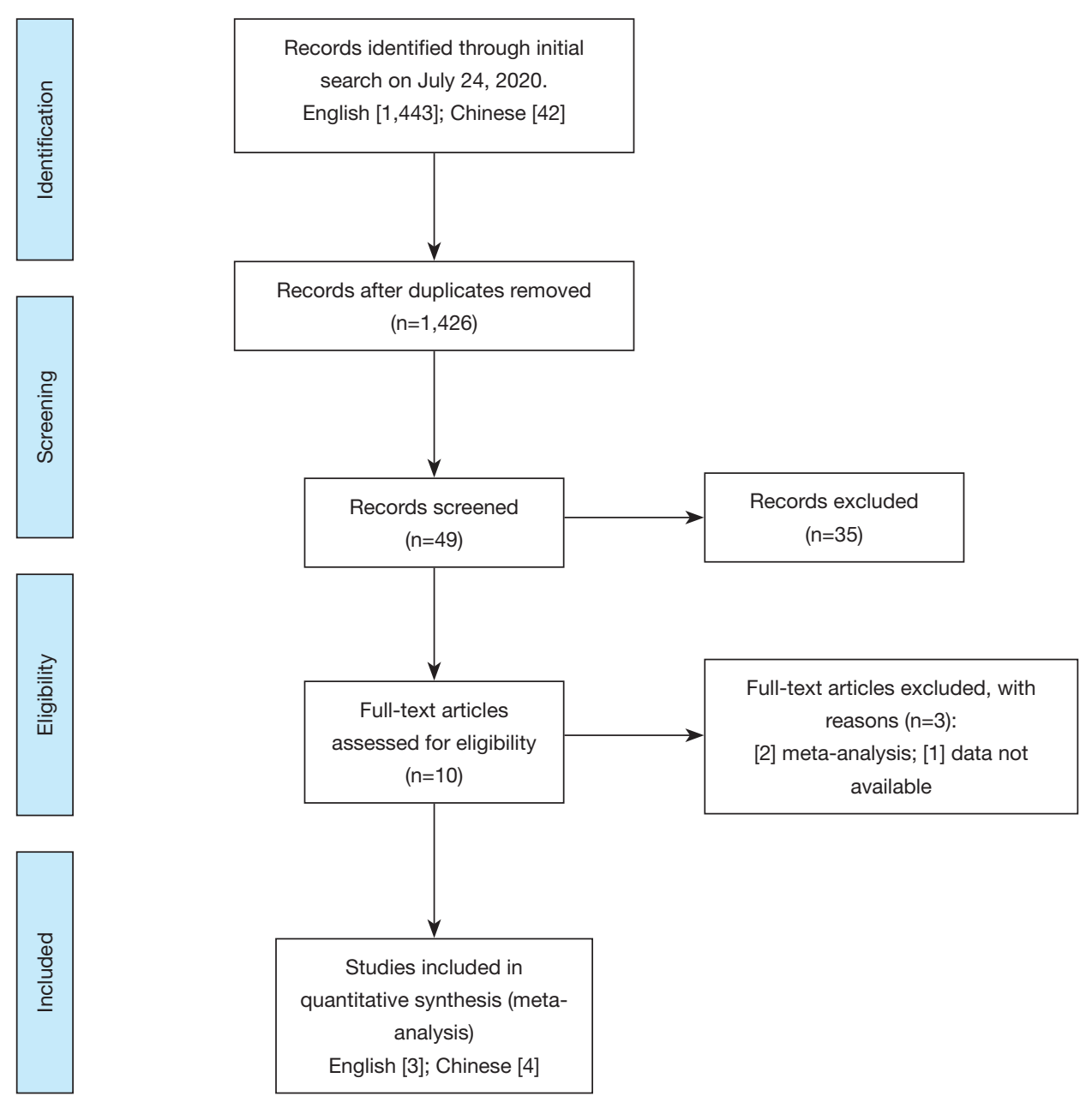

Figure 1 The flow chart of the study selection.

Besides, only two articles $(21,25)$ reported the results of high TG, and the pooled analysis indicated that patients with high TG were prone to high-stage $\mathrm{BC}$ with borderline statistical significance (RR: 1.74; 95\% CI: 1.00-3.01; $\mathrm{P}=0.05$ ) (Figure 2).

\section{MS and BC grade}

A total of six studies (19,21-25) reported the effect of MS on BC, and the pooled analysis indicated that MS can contribute substantially to the vulnerability of highgrade BC with significant difference (RR: $1.50 ; 95 \%$ CI: 1.37-1.65) (Figure 3). Furthermore, patients with obesity, hyperglycemia, hypertension, low HDL and high TG were at a higher risk of high-grade $\mathrm{BC}$ than their counterparts, and the corresponding RRs and 95\% CIs were 1.41 (1.18,
1.69), 1.42 (1.30, 1.56), 1.13 (1.03, 1.24), 1.29 (1.14, 1.46) and $1.28(1.11,1.46)$, respectively (Figure 3).

\section{Subgroup analysis of Chinese studies}

There were no significant changes in subgroup analysis of Chinese studies (22-25) except for the association between hypertension and BC grade (Supplementary material). We observed no significant difference between hypertension and BC grade (RR: 1.12, 95\% CI: 0.99-1.27).

\section{Discussion}

Currently, we observed that patients with MS presented a higher incidence of high-stage BC than those without MS. The similar effects were also observed in single MS 


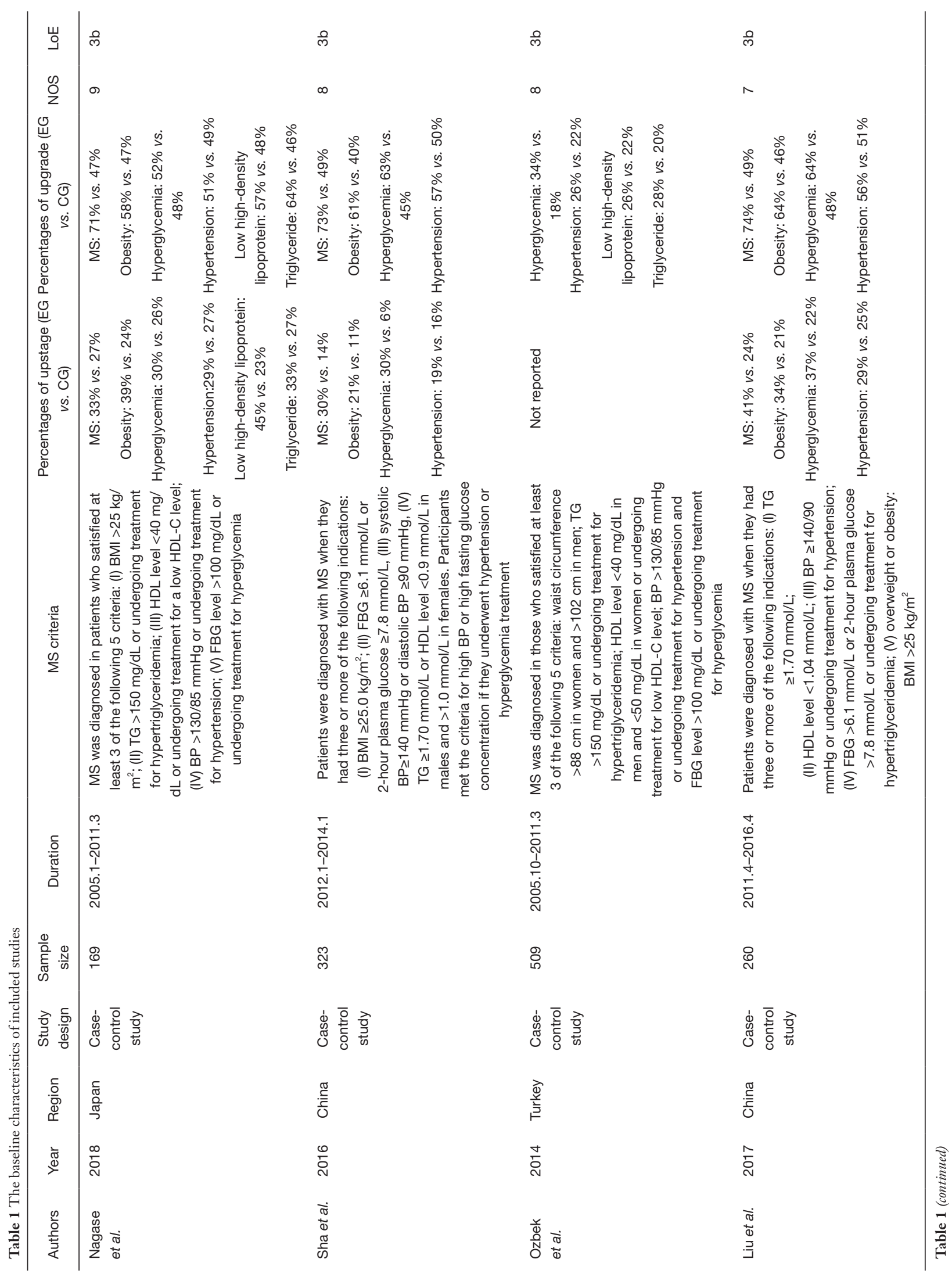




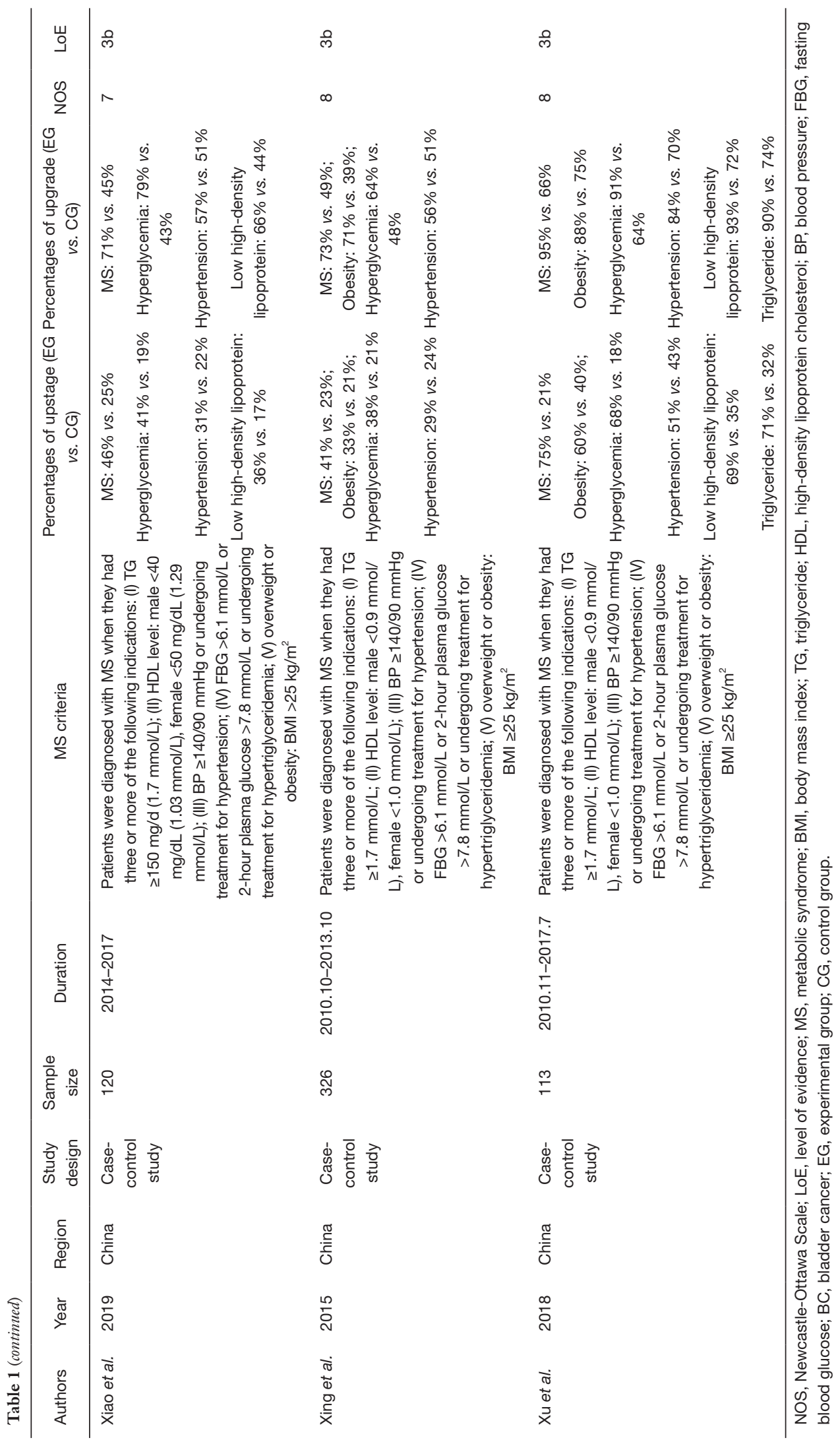


Obesity

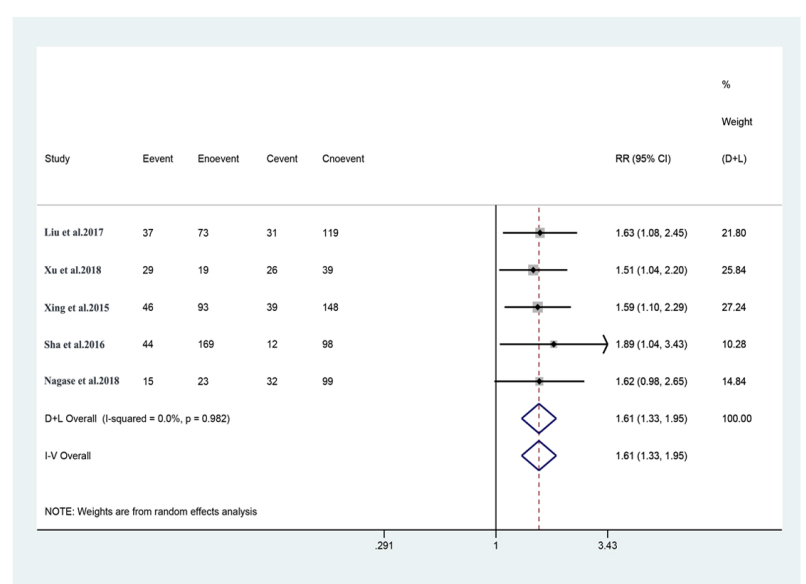

Hyperglycemia

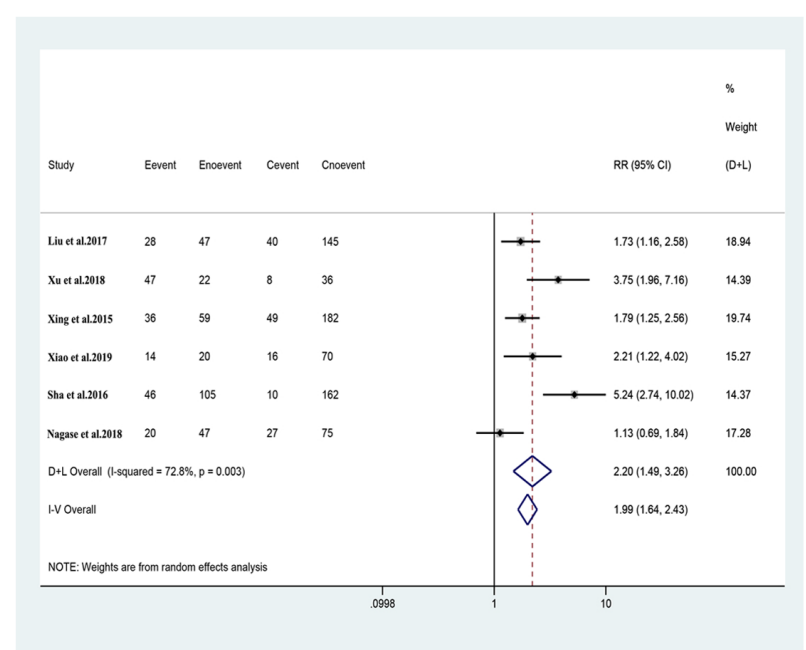

Low high-density lipoprotein

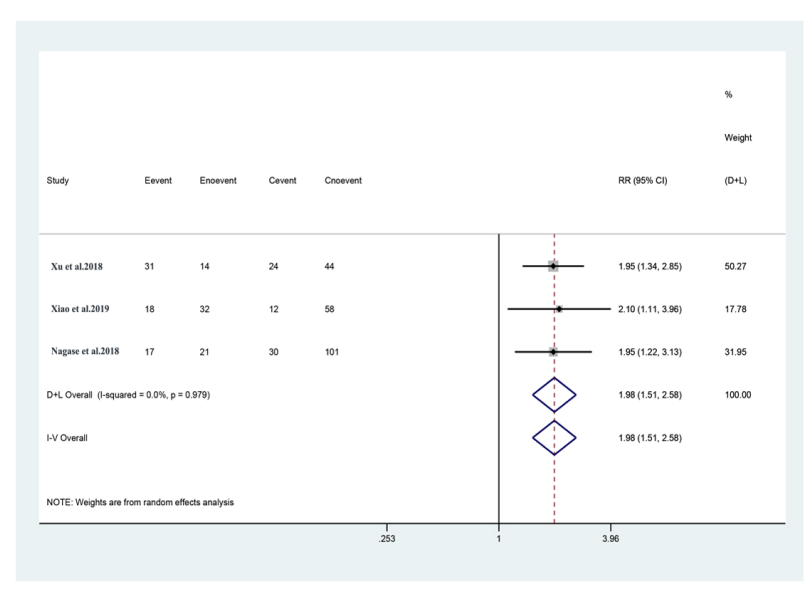

Metabolic syndrome

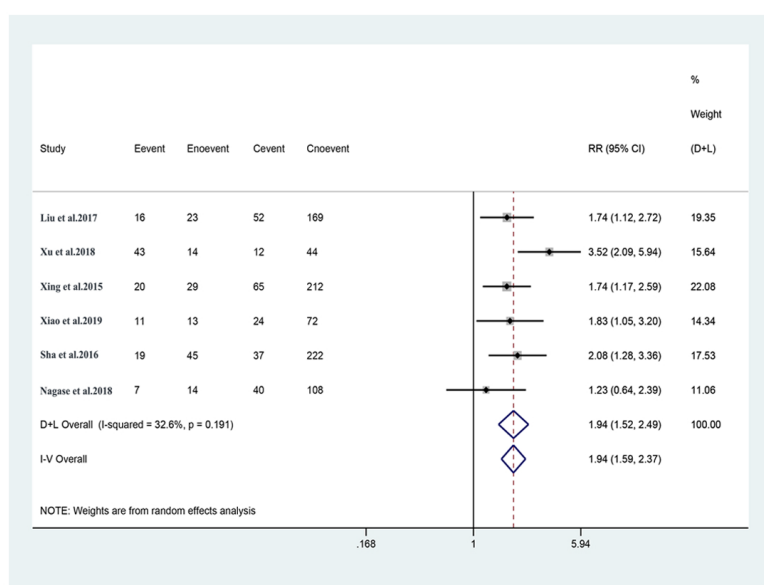

Hypertension

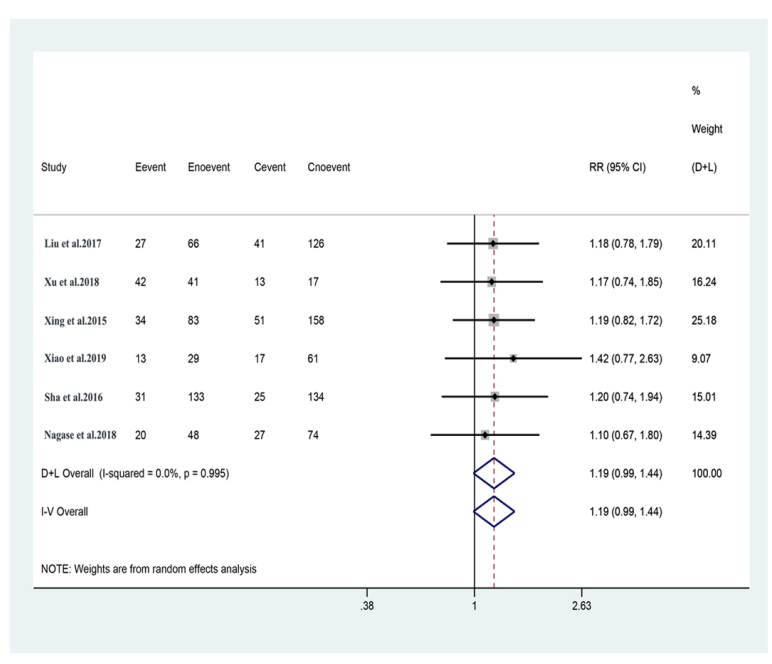

Triglyceride

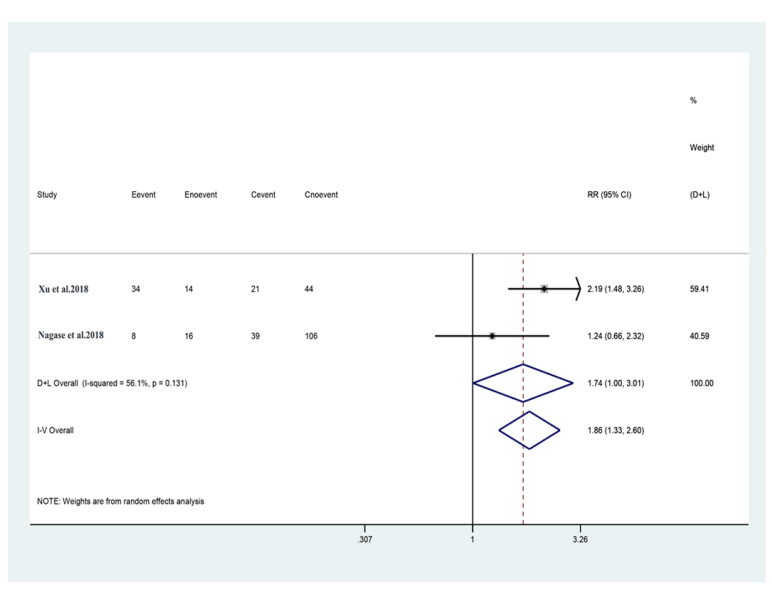

Figure 2 The meta-analysis results of metabolic syndrome and tumor stage of bladder cancer. 


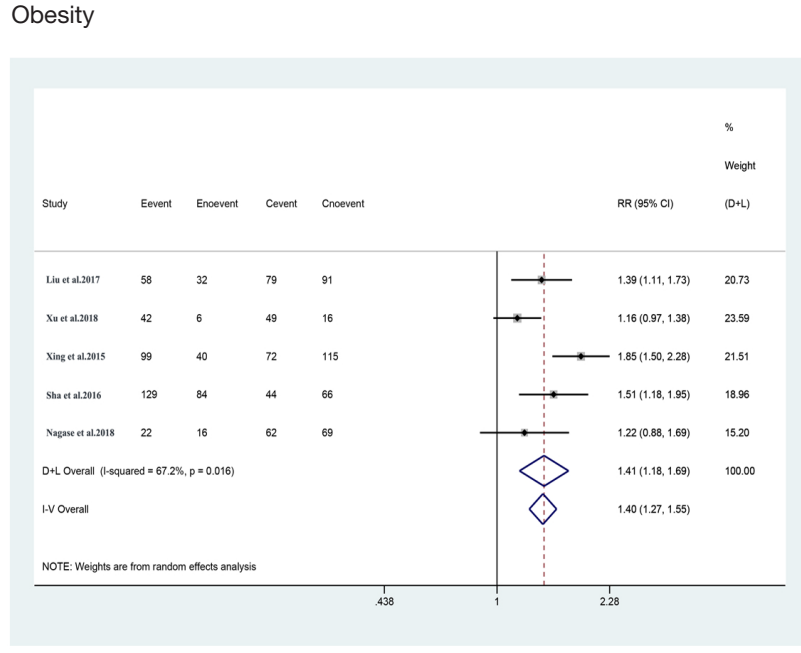

Hyperglycemia

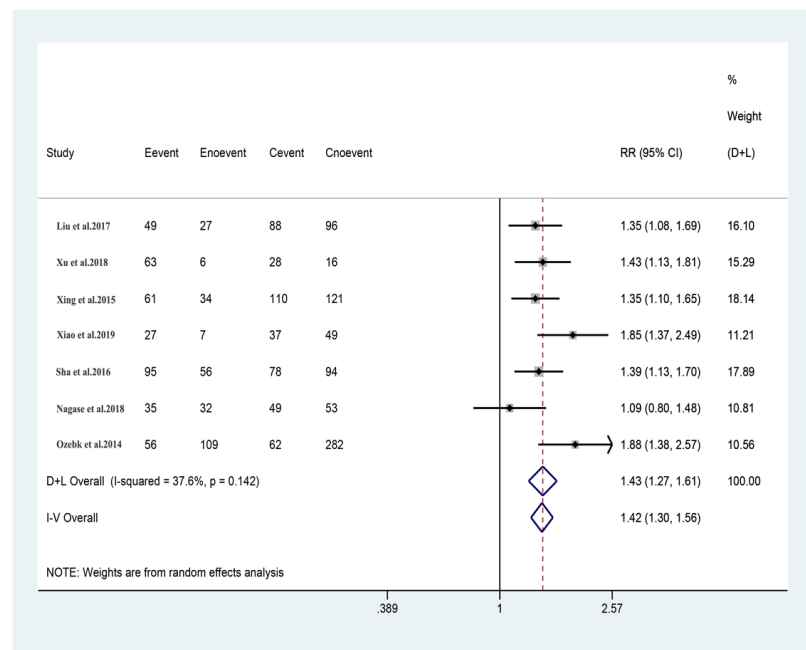

Low high-density lipoprotein

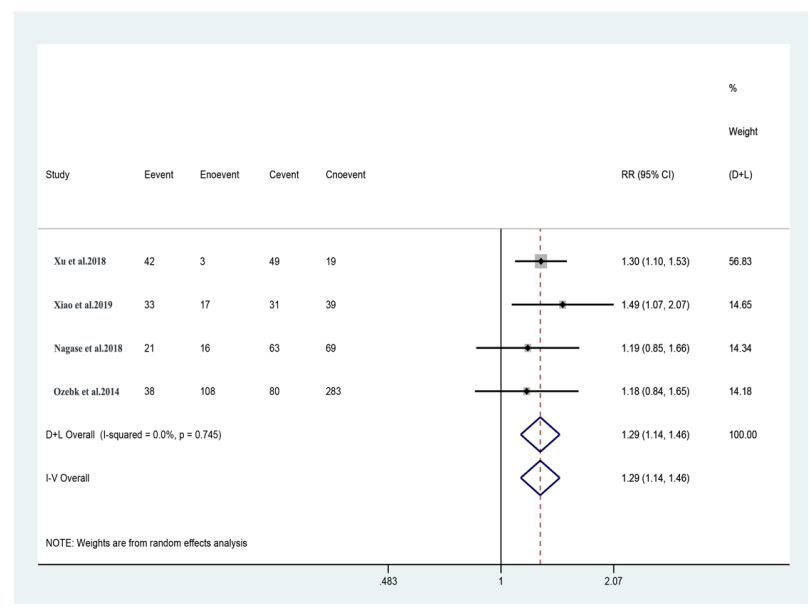

Metabolic syndrome

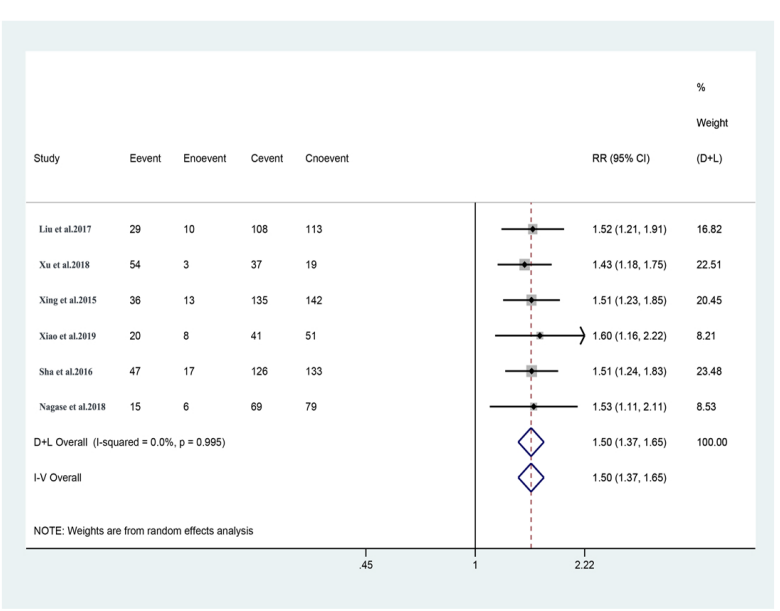

Hypertension

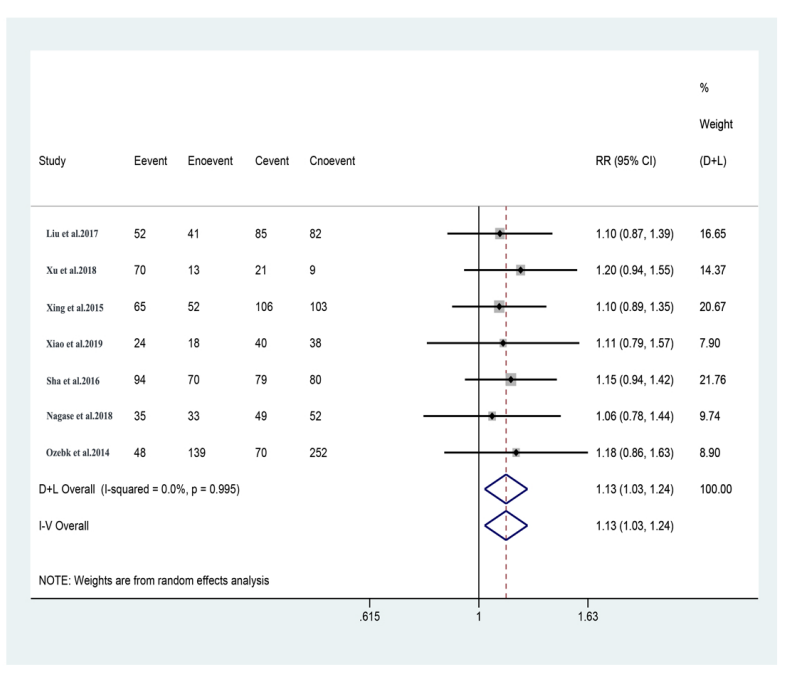

Triglyceride

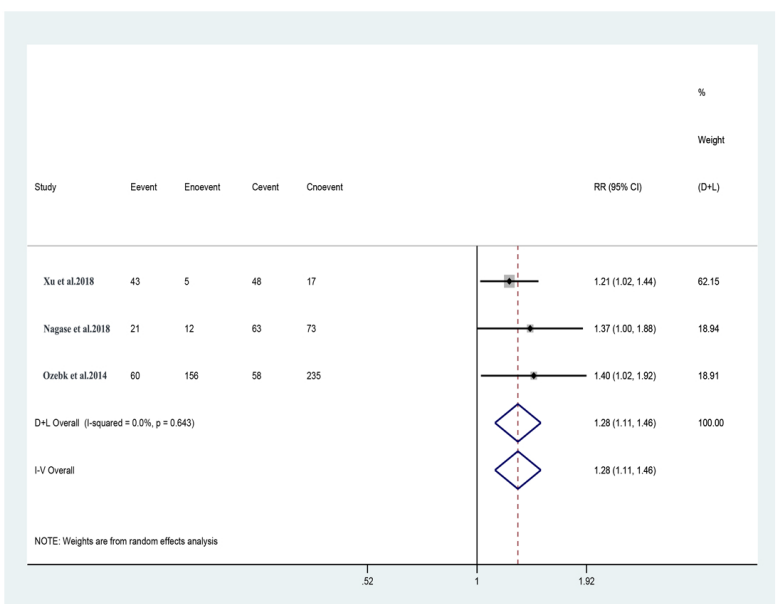

Figure 3 The meta-analysis results of metabolic syndrome and tumor grade of bladder cancer. 
parameters except for hypertension. In addition, MS and its components can contribute substantially to the vulnerability of high-grade $\mathrm{BC}$ with significant difference.

It is well-known that $\mathrm{BC}$ is a common and costly malignant tumor due to lifelong routine monitoring and treatment after diagnosis, and more importantly, some recurrence cases may develop into higher stage and grade, which brings about abundant burden to patients and medial resources (3). Similar to BC, MS is prone to affect older adults disproportionately with the highest prevalence in those aged 60 years and older (8). The previous meta-analyses focused on the association between BC susceptibility and the overall MS and its certain specific components, mainly involving in diabetes and excessive body weight (7,26-28). However, the impact of MS, as well as the components of MS, on the pathological features of $\mathrm{BC}$ remains controversial, and recently there have been publications exploring these correlations. A meta-analysis has been done under these circumstances.

As is well known, obesity and hyperglycemia represent two substantial components of MS, and these two metabolic conditions are highly correlated with each other. Thus, we assumed that the mechanisms of their carcinogenesis are similar and synergistic. The potential relationships between obesity and $\mathrm{BC}$ include the following three aspect: (I) insulin resistance and elevated serum level of insulinlike growth factor (IGF) -1 (29). IGF-1 might contribute to proliferation and restrain apoptosis, and eventually lead to cancer development and progression. (II) excess energy in the hosts can increase the risks of carcinogenesis (19); (III) Excess fat is also related to systemic inflammatory response, which might play a key part in cancer (19). As for the link between hyperglycemia and $\mathrm{BC}$, in addition to decreased insulin sensitivity and increased level of IGF-1, the possible reason is that hyperglycemia can cause dysfunction of the important cell signaling system regulated by the protein kinase $\mathrm{C}$ family, thus inducing tumor growth and metastasis (19).

So far, little is known about potential pathways between low HDL levels, hypertension, and malignant tumors. Both low HDL levels and hypertension play an important role in arteriosclerosis. Several studies indicated that hypertension itself is an important risk factor for malignant tumors $(30,31)$. Our study found that low HDL level was associated with upstaging and upgrading of $\mathrm{BC}$, and hypertension was related to high tumor grade. The potential mechanism of these findings necessitates to be further studied. At the same time, physicians should remember that low HDL levels and hypertension might worsen a patient's metabolic disorder and upstage and upgrade the $\mathrm{BC}$, and appropriate treatments should be provided to such patients (21).

A limitation of this analysis is that due to the study design, the studies included in this meta-analysis have inherent limitations. Retrospective study can contribute to selection bias, such as Berkson bias and Neyman bias, so we use RR to control bias and improve the reliability of analysis results and conclusions. Secondly, the broad heterogeneity of populations, designs and definitions of outcomes should be considered. For example, there were no accurate definitions of MS. Finally, the limited number of studies and sample size discouraged us from making definite conclusions.

Based on these data, MS should be studied within screening protocols to detect more aggressive tumors early; furthermore, MS could be included in current clinical nomograms to improve clinical staging and to personalize the treatment.

\section{Conclusions}

This meta-analysis revealed that MS and its components might be associated with high BC stage and grade. Further larger cohort studies are needed to confirm our findings.

\section{Acknowledgments}

Funding: This work was supported by Department of Science and Technology of Sichuan Province (2020YFH0099) and the National Natural Science Foundation of China (No. 81370272, 30901621/C1705). The funders had no role in study design, data collection or analysis, preparation of the manuscript, or the decision to publish.

\section{Footnote}

Reporting Checklist: The authors have completed the PRISMA reporting checklist. Available at http://dx.doi. org/10.21037/tcr-20-3350

Conflicts of Interest: All authors have completed the ICMJE uniform disclosure form (available at http://dx.doi. org/10.21037/tcr-20-3350). The authors have no conflicts of interest to declare.

Ethical Statement: The authors are accountable for all 
aspects of the work in ensuring that questions related to the accuracy or integrity of any part of the work are appropriately investigated and resolved.

Open Access Statement: This is an Open Access article distributed in accordance with the Creative Commons Attribution-NonCommercial-NoDerivs 4.0 International License (CC BY-NC-ND 4.0), which permits the noncommercial replication and distribution of the article with the strict proviso that no changes or edits are made and the original work is properly cited (including links to both the formal publication through the relevant DOI and the license). See: https://creativecommons.org/licenses/by-nc-nd/4.0/.

\section{References}

1. Sung H, Ferlay J, Siegel RL, et al. Global cancer statistics 2020: GLOBOCAN estimates of incidence and mortality worldwide for 36 cancers in 185 countries. CA Cancer J Clin 2021;71:209-49.

2. Han S, Zhang S, Chen W, et al. Analysis of the status and trends of bladder cancer incidence in China. Oncol Prog 2013;11:89-95.

3. Leal J, Luengo-Fernandez R, Sullivan R, et al. Economic Burden of Bladder Cancer Across the European Union. Eur Urol 2016;69:438-47.

4. Guha N, Steenland NK, Merletti F, et al. Bladder cancer risk in painters: a meta-analysis. Occup Environ Med 2010;67:568-73

5. Bayne CE, Farah D, Herbst KW, et al. Role of urinary tract infection in bladder cancer: a systematic review and meta-analysis. World J Urol 2018;36:1181-90.

6. Akhtar S, Al-Shammari A, Al-Abkal J. Chronic urinary tract infection and bladder carcinoma risk: a metaanalysis of case-control and cohort studies. World J Urol 2018;36:839-48.

7. Peng XF, Meng XY, Wei C, et al. The association between metabolic syndrome and bladder cancer susceptibility and prognosis: an updated comprehensive evidence synthesis of 95 observational studies involving 97,795,299 subjects. Cancer Manag Res 2018;10:6263-74.

8. Ford ES, Giles WH, Dietz WH. Prevalence of the metabolic syndrome among US adults: findings from the third National Health and Nutrition Examination Survey. JAMA 2002;287:356-9.

9. Aguilar M, Bhuket T, Torres S, et al. Prevalence of the metabolic syndrome in the United States, 2003-2012. JAMA 2015;313:1973-4.
10. Gu D, Reynolds K, Wu X, et al. Prevalence of the metabolic syndrome and overweight among adults in China. Lancet 2005;365:1398-405.

11. Xi B, He D, Hu Y, et al. Prevalence of metabolic syndrome and its influencing factors among the Chinese adults: the China Health and Nutrition Survey in 2009. Prev Med 2013;57:867-71.

12. Stocks T, Bjørge T, Ulmer H, et al. Metabolic risk score and cancer risk: pooled analysis of seven cohorts. Int $\mathrm{J}$ Epidemiol 2015;44:1353-63.

13. Gacci M, Russo GI, De Nunzio C, et al. Meta-analysis of metabolic syndrome and prostate cancer. Prostate Cancer Prostatic Dis 2017;20:146-55.

14. Moher D, Liberati A, Tetzlaff J, et al. Preferred reporting items for systematic reviews and meta-analyses: the PRISMA statement. PLoS Med 2009;6:e1000097.

15. Wells GA, Shea B, O'Connell D, et al. The NewcastleOttawa Scale (NOS) for assessing the quality if nonrandomized studies in meta-analyses. Ottawa Hosp Res Inst. Available online: http://www.ohri.ca/programs/clinical_ epidemiology/oxford.asp. Accessed August 10, 2020.

16. Evidence-Based Cf. Oxford Centre for evidence-based medicine: levels of evidence, 2009. Available online: http:// www.cebm.net/oxford-centreevidence-based-medicinelevels-evidence-march-2009/

17. Higgins JP, Thompson SG, Deeks JJ, et al. Measuring inconsistency in meta-analyses. BMJ 2003;327:557-60.

18. Higgins JP, Thompson SG. Quantifying heterogeneity in a meta-analysis. Stat Med 2002;21:1539-58.

19. Sha $\mathrm{N}, \mathrm{Xu} \mathrm{H}$, Chen $\mathrm{T}$, et al. The evaluation of the association between the metabolic syndrome and tumor grade and stage of bladder cancer in a Chinese population. Onco Targets Ther 2016;9:1175-9.

20. Ozbek E, Otunctemur A, Dursun M, et al. Association between the metabolic syndrome and high tumor grade and stage of primary urothelial cell carcinoma of the bladder. Asian Pac J Cancer Prev 2014;15:1447-51.

21. Nagase K, Tobu S, Kusano S, et al. The Association between Metabolic Syndrome and High-Stage Primary Urothelial Carcinoma of the Bladder. Curr Urol 2018;12:39-42.

22. Liu Q, Li J, Qu W, et al. The association of metabolic syndrome and its components with tumor stage and grade of primary bladder carcinoma. Modern Oncol 2017;(15):2449-51.

23. Xiao R, Yu J, Xu J, et al. Association of metabolic syndrome and its components with tumor stage and grade of bladder cancer. Electronic Journal of Clinical Medical 
Literature 2019;6:25-6.

24. Xing Q, Guo Z, Li C, et al. Correlation of metabolic syndrome with tumor grade and stage of primary bladder carcinoma. Chin J Urol 2015;36:761-4.

25. Xu J, Zhuo Y, Guo Z, et al. Clinical correlation between the metabolic syndrome and tumor grade and stage in elderly male patients with bladder cancer. Journal of Jinan University (Natural Science \& Medicine Edition) 2018;39:529-34.

26. Lin Y, Wang Y, Wu Q, et al. Association between obesity and bladder cancer recurrence: A meta-analysis. Clin Chim Acta 2018;480:41-6.

27. Yang XQ, Xu C, Sun Y, et al. Diabetes mellitus increases the risk of bladder cancer: an updated meta-analysis. Asian
Pac J Cancer Prev 2013;14:2583-9.

28. Qin Q, Xu X, Wang X, et al. Obesity and risk of bladder cancer: a meta-analysis of cohort studies. Asian Pac J Cancer Prev 2013;14:3117-21.

29. Hursting SD, Hursting MJ. Growth signals, inflammation, and vascular perturbations: mechanistic links between obesity, metabolic syndrome, and cancer. Arterioscler Thromb Vasc Biol 2012;32:1766-70.

30. Schmieder RE, Delles C, Messerli FH. Diuretic therapy and the risk for renal cell carcinoma. J Nephrol 2000;13:343-6.

31. Wannamethee G, Shaper AG. Blood pressure and cancer in middle-aged British men. Int J Epidemiol 1996;25:22-31.
Cite this article as: Feng D, Song P, Yang Y, Wei W, Li L. Is metabolic syndrome associated with high tumor grade and stage of bladder cancer: a systematic review and meta-analysis. Transl Cancer Res 2021;10(5):2188-2198. doi: 10.21037/tcr-20-3350 


\section{Subject headings and text words related to metabolic syndrome and bladder cancer}

PubMed

Metabolic Syndrome (Mesh term)

Metabolic Syndromes

Syndrome, Metabolic

Syndromes, Metabolic

Metabolic Syndrome X

Insulin Resistance Syndrome X

Syndrome X, Metabolic

Syndrome X, Insulin Resistance

Metabolic X Syndrome

Syndrome, Metabolic X

$\mathrm{X}$ Syndrome, Metabolic

Dysmetabolic Syndrome X

Syndrome X, Dysmetabolic

Reaven Syndrome X

Syndrome X, Reaven

Metabolic Cardiovascular Syndrome

Cardiovascular Syndrome, Metabolic

Cardiovascular Syndromes, Metabolic

Syndrome, Metabolic Cardiovascular

Urinary Bladder Neoplasms (Mesh term)

Neoplasm, Urinary Bladder

Urinary Bladder Neoplasm

Neoplasms, Bladder

Bladder Neoplasms

Bladder Neoplasm

Neoplasm, Bladder

Bladder Tumors

Bladder Tumor

Tumor, Bladder

Tumors, Bladder

Urinary Bladder Cancer

Cancer, Urinary Bladder

Malignant Tumor of Urinary Bladder

Cancer of the Bladder

Bladder Cancer

Bladder Cancers

Cancer, Bladder

Cancer of Bladder

Search strategy

PubMed

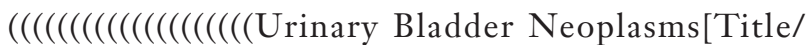

Abstract]) OR (Neoplasm, Urinary Bladder[Title/
Abstract])) OR (Urinary Bladder Neoplasm[Title/ Abstract])) OR (Neoplasms, Bladder[Title/Abstract])) OR (Bladder Neoplasms[Title/Abstract])) OR (Bladder Neoplasm[Title/Abstract])) OR (Neoplasm, Bladder[Title/ Abstract])) OR (Bladder Tumors[Title/Abstract])) OR (Bladder Tumor[Title/Abstract])) OR (Tumor, Bladder[Title/Abstract])) OR (Tumors, Bladder[Title/ Abstract])) OR (Urinary Bladder Cancer[Title/Abstract])) ) OR (Cancer, Urinary Bladder[Title/Abstract])) OR (Malignant Tumor of Urinary Bladder[Title/ Abstract])) OR (Cancer of the Bladder[Title/Abstract])) OR (Bladder Cancer[Title/Abstract])) OR (Bladder Cancers[Title/Abstract])) OR (Cancer, Bladder[Title/ Abstract])) OR (Cancer of Bladder[Title/Abstract])) AND $((()((()(()((()((($ Metabolic Syndrome[Title/Abstract]) OR (Metabolic Syndromes[Title/Abstract])) OR (Syndrome, Metabolic[Title/Abstract])) OR (Syndromes, Metabolic[Title/Abstract])) OR (Metabolic Syndrome $\mathrm{X}$ [Title/Abstract])) OR (Insulin Resistance Syndrome X[Title/Abstract])) OR (Syndrome X, Metabolic[Title/ Abstract])) OR (Syndrome X, Insulin Resistance[Title/ Abstract])) OR (Metabolic X Syndrome[Title/Abstract])) OR (Syndrome, Metabolic X[Title/Abstract])) OR (X Syndrome, Metabolic[Title/Abstract])) OR (Dysmetabolic Syndrome X[Title/Abstract])) OR (Syndrome X, Dysmetabolic[Title/Abstract])) OR (Reaven Syndrome X[Title/Abstract])) OR (Syndrome X, Reaven[Title/ Abstract])) OR (Metabolic Cardiovascular Syndrome[Title/ Abstract])) OR (Cardiovascular Syndrome, Metabolic[Title/ Abstract])) OR (Cardiovascular Syndromes, Metabolic[Title/Abstract])) OR (Syndrome, Metabolic Cardiovascular[Title/Abstract])) 31 records

The Cochrane Library and Embase through OVID.

\#1. (Metabolic Syndrome or Metabolic Syndromes or Syndrome, Metabolic or Syndromes, Metabolic or Metabolic Syndrome X or Insulin Resistance Syndrome $\mathrm{X}$ or Syndrome X, Metabolic or Syndrome X, Insulin Resistance or Metabolic X Syndrome or Syndrome, Metabolic X or X Syndrome, Metabolic or Dysmetabolic Syndrome X or Syndrome X, Dysmetabolic or Reaven Syndrome X or Syndrome X, Reaven or Metabolic Cardiovascular Syndrome or Cardiovascular Syndrome, Metabolic or Cardiovascular Syndromes, Metabolic or Syndrome, Metabolic Cardiovascular).kw. 26753 records

\#2. (Urinary Bladder Neoplasms or Neoplasm, Urinary Bladder or Urinary Bladder Neoplasm or Neoplasms, Bladder or Bladder Neoplasms or Bladder Neoplasm or 
Neoplasm, Bladder or Bladder Tumors or Bladder Tumor or Tumor, Bladder or Tumors, Bladder or Urinary Bladder Cancer or Cancer, Urinary Bladder or Malignant Tumor of Urinary Bladder or Cancer of the Bladder or Bladder Cancer or Bladder Cancers or Cancer, Bladder or Cancer of Bladder).kw 18867 records.

\#3 \# 1 and \# 2.10 records.

Web of Science

\#1. Topic: (Metabolic Syndrome) OR Topic: (Metabolic Syndromes) OR Topic: (Syndrome, Metabolic) OR Topic: (Syndromes, Metabolic) OR Topic: (Metabolic Syndrome X) OR Topic: (Insulin Resistance Syndrome X) OR Topic: (Syndrome X, Metabolic) OR Topic: (Syndrome X, Insulin Resistance) OR Topic: (Metabolic X Syndrome) OR Topic: (Syndrome, Metabolic X) OR Topic: (X Syndrome, Metabolic) OR Topic: (Dysmetabolic Syndrome X) OR Topic: (Syndrome X, Dysmetabolic) OR Topic: (Reaven Syndrome X) OR Topic: (Syndrome X, Reaven) OR Topic: (Metabolic Cardiovascular Syndrome) OR Topic: (Cardiovascular Syndrome, Metabolic) OR Topic: (Cardiovascular Syndromes, Metabolic) OR Topic:
(Syndrome, Metabolic Cardiovascular) 294,329 records \#2. Topic: (Urinary Bladder Neoplasms) OR Topic: (Neoplasm, Urinary Bladder) OR Topic: (Urinary Bladder Neoplasm) OR Topic: (Neoplasms, Bladder) OR Topic: (Bladder Neoplasms) OR Topic: (Bladder Neoplasm) OR Topic: (Neoplasm, Bladder) OR Topic: (Bladder Tumors) OR Topic: (Bladder Tumor) OR Topic: (Tumor, Bladder) OR Topic: (Tumors, Bladder) OR Topic: (Urinary Bladder Cancer) OR Topic: (Cancer, Urinary Bladder) OR Topic: (Malignant Tumor of Urinary Bladder) OR Topic: (Cancer of the Bladder) OR Topic: (Bladder Cancer) OR Topic: (Bladder Cancers) OR Topic: (Cancer, Bladder) OR Topic: (Cancer of Bladder) 160,137 records.

\#3 \#1 and \#2 1402 records

Chinese

CNKI: 23 records

WANGFANG: 9 records

VIP: 10 records

Subgroup analysis of Chinese studies

Metabolic syndrome (MS) and bladder cancer (BC) stage 


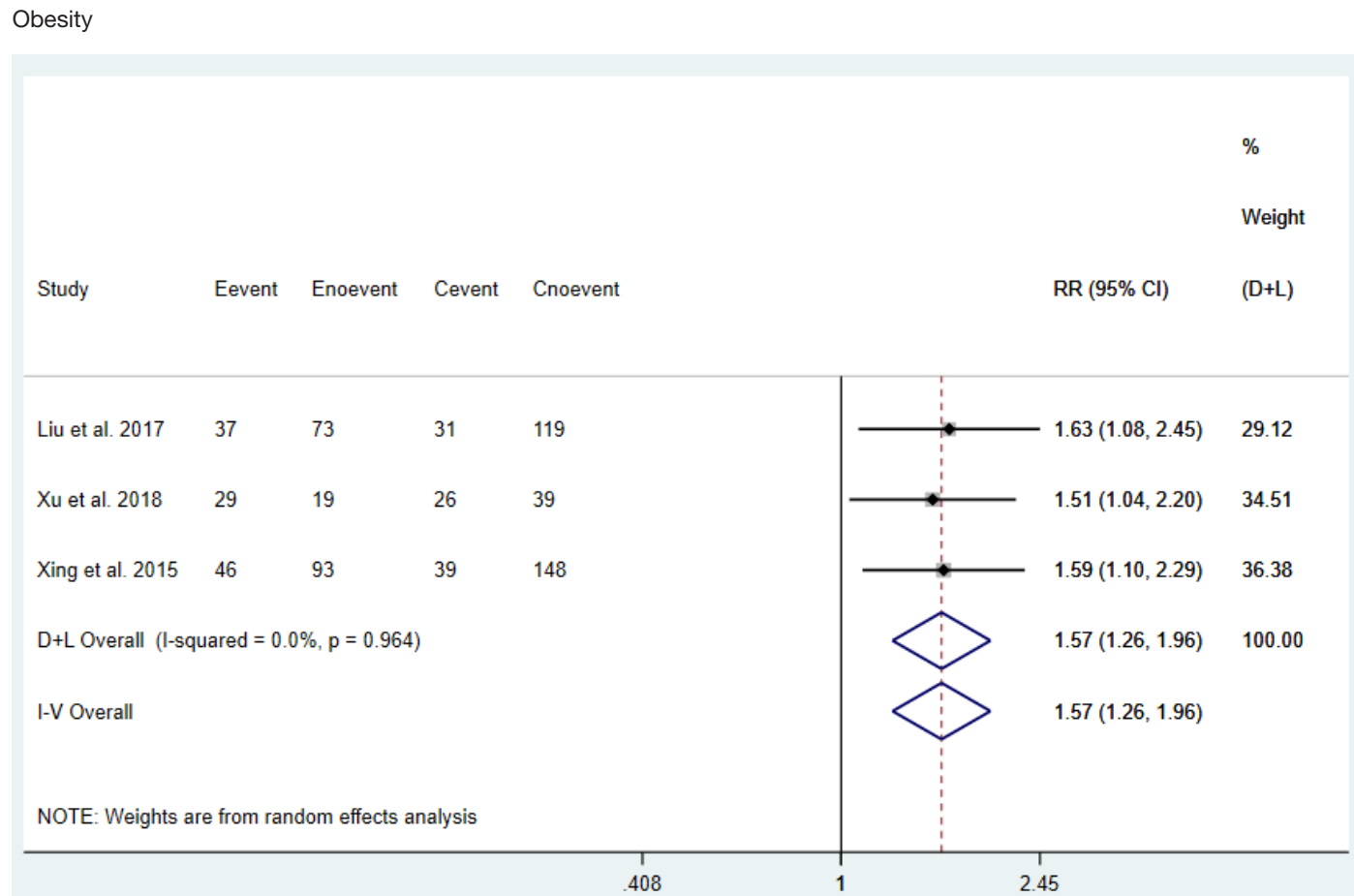

MS

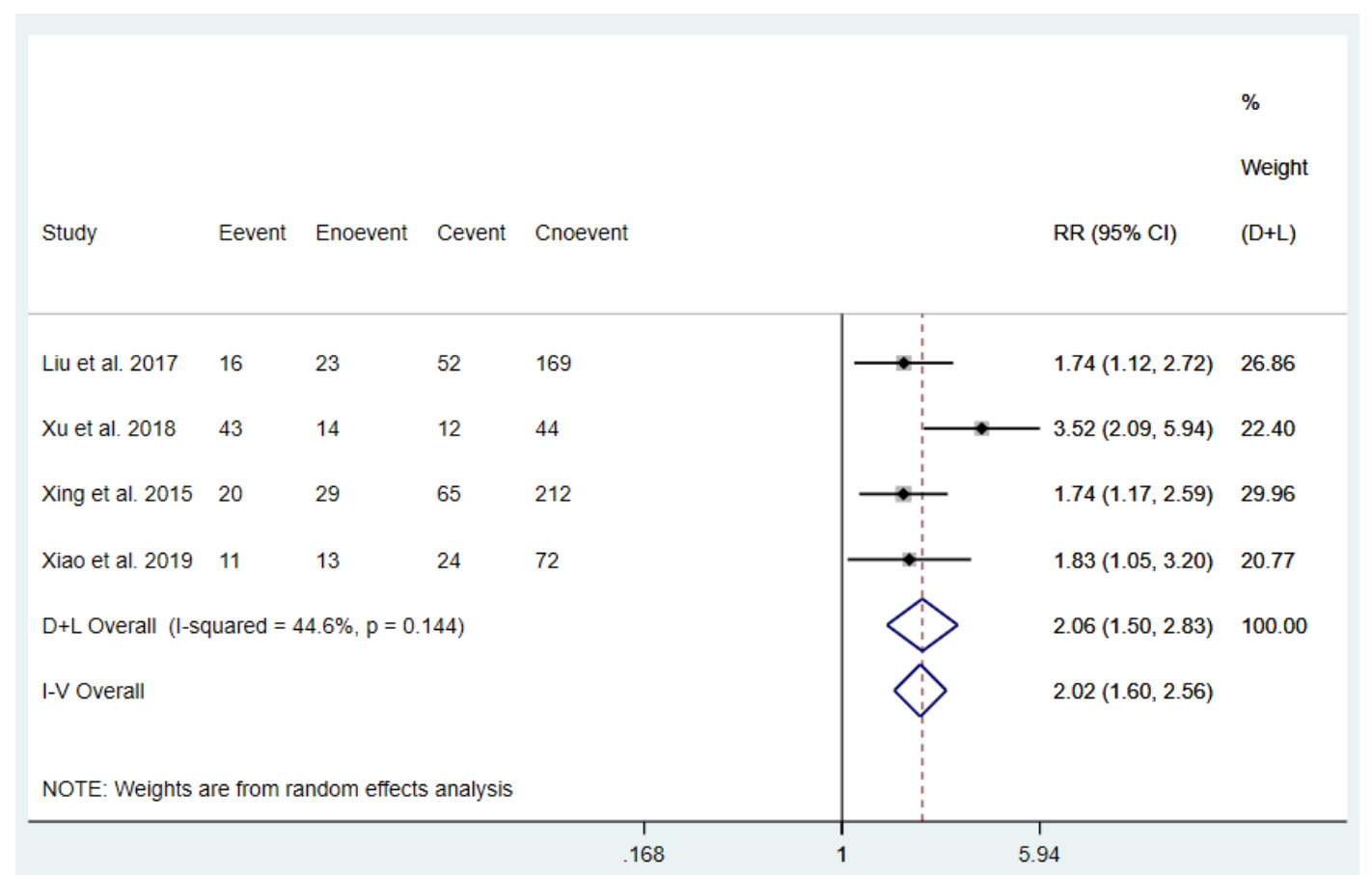




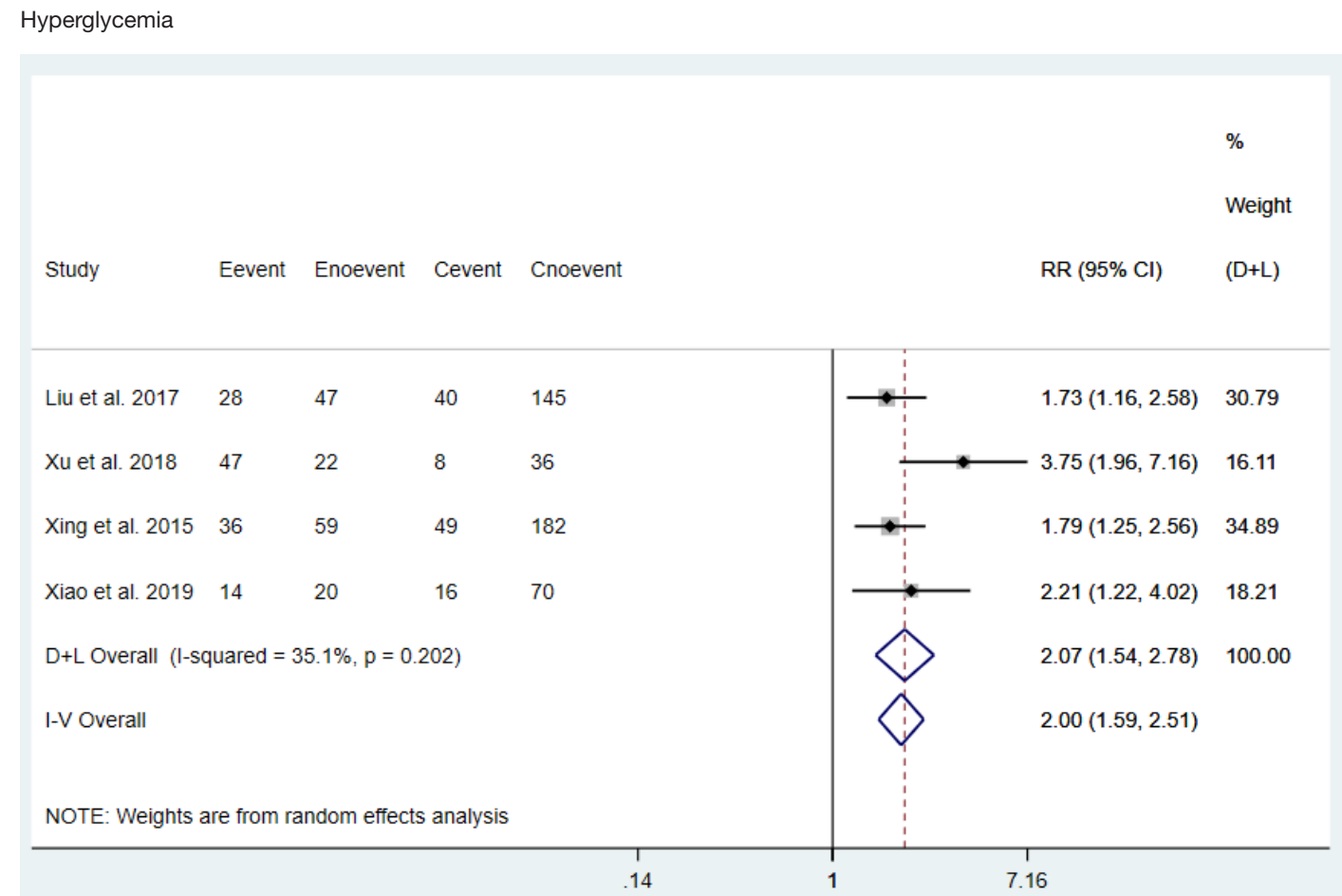

\begin{tabular}{|c|c|c|c|c|c|c|}
\hline \multirow[b]{2}{*}{ Study } & \multirow[b]{2}{*}{ Eevent } & \multirow[b]{2}{*}{ Enoevent } & \multirow[b]{2}{*}{ Cevent } & \multirow[b]{2}{*}{ Cnoevent } & \multirow[b]{2}{*}{$\mathrm{RR}(95 \% \mathrm{Cl})$} & $\begin{array}{l}\% \\
\text { Weight }\end{array}$ \\
\hline & & & & & & $(\mathrm{D}+\mathrm{L})$ \\
\hline Liu et al. 2017 & 27 & 66 & 41 & 126 & $1.18(0.78,1.79)$ & 28.49 \\
\hline Xu et al. 2018 & 42 & 41 & 13 & 17 & $1.17(0.74,1.85)$ & 23.00 \\
\hline Xing et al. 2015 & 34 & 83 & 51 & 158 & $1.19(0.82,1.72)$ & 35.67 \\
\hline Xiao et al. 2019 & 13 & 29 & 17 & 61 & $1.42(0.77,2.63)$ & 12.84 \\
\hline \multicolumn{5}{|c|}{$D+L$ Overall $(\mathrm{I}$-squared $=0.0 \%, p=0.960$ ) } & $1.21(0.97,1.51)$ & 100.00 \\
\hline I-V Overall & & & & & $1.21(0.97,1.51)$ & \\
\hline \multicolumn{7}{|c|}{ NOTE: Weights are from random effects analysis } \\
\hline & & & & .38 & 63 & \\
\hline
\end{tabular}




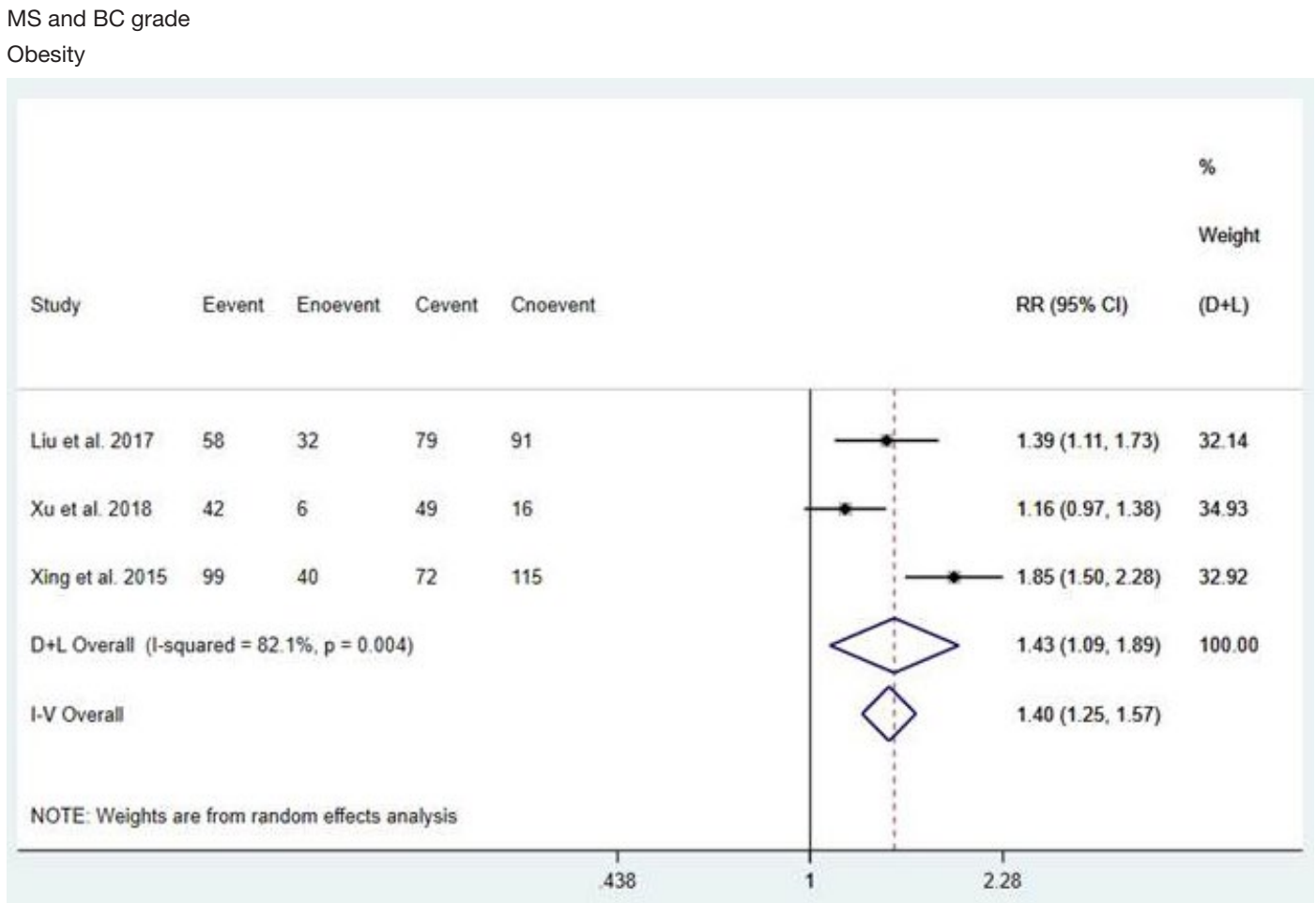

MS

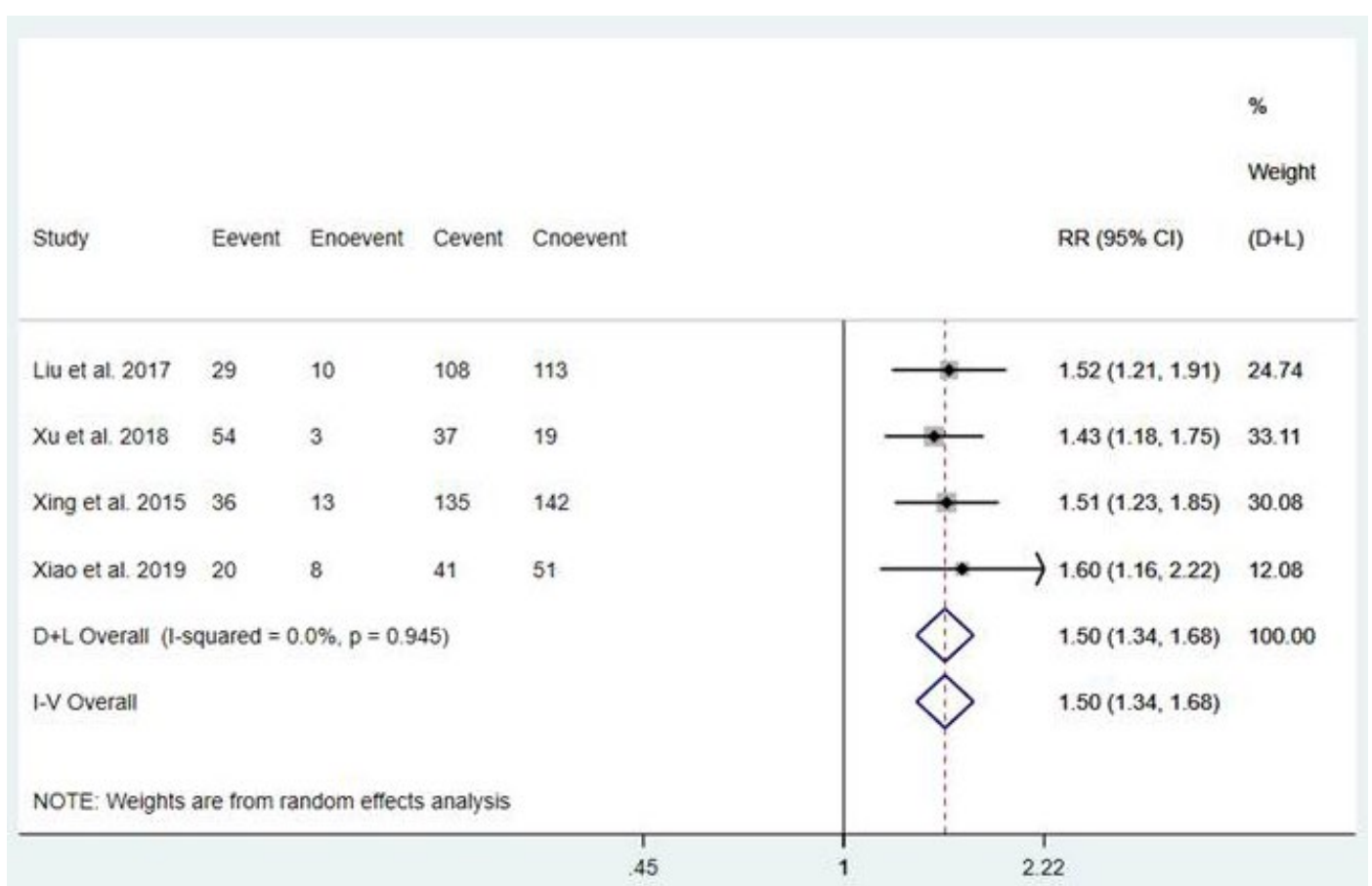




\begin{tabular}{|c|c|c|c|c|c|c|}
\hline \multirow[b]{2}{*}{ Study } & \multirow[b]{2}{*}{ Eevent } & \multirow[b]{2}{*}{ Enoevent } & \multirow[b]{2}{*}{ Cevent } & \multirow[b]{2}{*}{ Cnoevent } & \multirow[b]{2}{*}{$\mathrm{RR}(95 \% \mathrm{Cl})$} & \multirow{2}{*}{$\begin{array}{l}\% \\
\text { Weight } \\
(D+L)\end{array}$} \\
\hline & & & & & & \\
\hline Liu et al. 2017 & 49 & 27 & 88 & 96 & $1.35(1.08,1.69)$ & 26.77 \\
\hline Xu et al. 2018 & 63 & 6 & 28 & 16 & $1.43(1.13,1.81)$ & 24.82 \\
\hline Xing et al. 2015 & 61 & 34 & 110 & 121 & $1.35(1.10,1.65)$ & 32.18 \\
\hline Xiao et al. 2019 & 27 & 7 & 37 & 49 & $1.85(1.37,2.49)$ & 16.23 \\
\hline \multicolumn{5}{|c|}{$D+L$ Overall $(I-$ squared $=12.1 \%, p=0.332)$} & $1.44(1.27,1.63)$ & 100.00 \\
\hline \multicolumn{5}{|l|}{ I-V Overall } & \multicolumn{2}{|l|}{$1.44(1.28,1.61)$} \\
\hline \multicolumn{7}{|c|}{ NOTE: Weights are from random effects analysis } \\
\hline & & & & 40 & 2.49 & \\
\hline
\end{tabular}

\begin{tabular}{|c|c|c|c|c|c|c|}
\hline \multirow[b]{2}{*}{ Study } & \multirow[b]{2}{*}{ Eevent } & \multirow[b]{2}{*}{ Enoevent } & \multirow[b]{2}{*}{ Cevent } & \multirow[b]{2}{*}{ Cnoevent } & \multirow[b]{2}{*}{$\mathrm{RR}(95 \% \mathrm{Cl})$} & $\begin{array}{l}\% \\
\text { Weight }\end{array}$ \\
\hline & & & & & & $(\mathrm{D}+\mathrm{L})$ \\
\hline Liu et al. 2017 & 52 & 41 & 85 & 82 & $1.10(0.87,1.39)$ & 27.95 \\
\hline Xu et al. 2018 & 70 & 13 & 21 & 9 & $1.20(0.94,1.55)$ & 24.11 \\
\hline Xing et al. 2015 & 65 & 52 & 106 & 103 & $1.10(0.89,1.35)$ & 34.68 \\
\hline Xiao et al. 2019 & 24 & 18 & 40 & 38 & $1.11(0.79,1.57)$ & 13.26 \\
\hline \multicolumn{5}{|c|}{$\mathrm{D}+\mathrm{L}$ Overall $(\mathrm{I}$-squared $=0.0 \%, \mathrm{p}=0.943$ ) } & $1.12(0.99,1.27)$ & 100.00 \\
\hline I-V Overall & & & & & $1.12(0.99,1.27)$ & \\
\hline \multicolumn{7}{|c|}{ NOTE: Weights are from random effects analysis } \\
\hline & & & & .639 & 57 & \\
\hline
\end{tabular}

